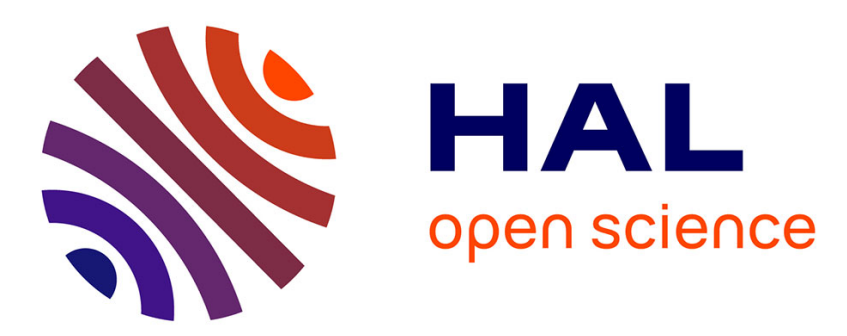

\title{
How metallic are noble-metal clusters? Static screening and polarizability in quantum-sized silver and gold nanoparticles
}

Rajarshi Sinha-Roy, Pablo García-González, Hans-Christian Weissker

\section{- To cite this version:}

Rajarshi Sinha-Roy, Pablo García-González, Hans-Christian Weissker. How metallic are noble-metal clusters? Static screening and polarizability in quantum-sized silver and gold nanoparticles. Nanoscale, 2020, 12 (7), pp.4452-4458. 10.1039/C9NR08608K . hal-02495773

\section{HAL Id: hal-02495773 \\ https://hal.science/hal-02495773}

Submitted on 2 Mar 2020

HAL is a multi-disciplinary open access archive for the deposit and dissemination of scientific research documents, whether they are published or not. The documents may come from teaching and research institutions in France or abroad, or from public or private research centers.
L'archive ouverte pluridisciplinaire HAL, est destinée au dépôt et à la diffusion de documents scientifiques de niveau recherche, publiés ou non, émanant des établissements d'enseignement et de recherche français ou étrangers, des laboratoires publics ou privés. 


\section{How Metallic are Noble-Metal Clusters? Static} Screening and Polarizability in Quantum-Sized

\section{Silver and Gold Nanoparticles}

Rajarshi Sinha-Roy, ${ }^{*, \dagger, \ddagger}$ Pablo García-González, ${ }^{\ddagger}$ đand Hans-Christian

$$
\text { Weissker }{ }^{*, \dagger}, \uparrow
$$

$\dagger$ †ix-Marseille Univ., CNRS, CINAM, Marseille, France.

$\ddagger$ Departamento de Física Teórica de la Materia Condensada and Condensed Matter Physics Center (IFIMAC), Universidad Autónoma de Madrid, E-28049 Madrid, Spain \European Theoretical Spectroscopy Facility (ETSF)

E-mail: sinharoy@cinam.univ-mrs.fr; weissker@cinam.univ-mrs.fr 


\begin{abstract}
Metallicity of nanoparticles can be defined in different ways. One possibility is to look at the degree to which external fields are screened inside the object. This screening would be complete in a classical perfect metal where surface charges arrange on the classical - i.e., abrupt - surface such that no internal fields exist. However, it is obvious that this situation is modified for very small clusters: the surface charges are "smeared out" at the surface, and the screening might be less complete. In the present work we ask the question as to how close small noblemetal clusters are to a classical metal. We show that, indeed, the screening is almost complete $(\approx 96 \%)$ already for as little as one atomic layer of the coinage metals, silver and gold alike. At the same time, we show that quantum effects, viz., electronic shell closings and the Friedel-like oscillations of the density, play a role, meaning that the clusters cannot be described solely using the concept of screening in a classical metal.
\end{abstract}

\title{
Keywords
}

Meallicity, static polarization, induced density, screening, DFT, noble-metal clusters, nanoparticles

\section{Introduction}

The long-standing question as to what is a metal receives different answers in different contexts and different communities. The existence of continuous electronic bands cutting through the Fermi surface is not an applicable criterion for small clusters because their electronic structure is necessarily made up of discrete states. By contrast, the screening of external fields by the distribution of induced electronic densities can be considered.

A classical metal does not support an internal electric field because the charge will arrange in such a way as to screen any external electric field $\mathbf{E}_{\text {ext }}(\mathbf{r})$. This makes it likewise impossible for a metallic object to have a non-zero permanent electric dipole 
moment, as this would imply a non-vanishing internal electric field. ${ }^{1,2}$ This has led to a number of studies, theoretical and experimental, that determine the dipole moment of small clusters. ${ }^{3-5}$ These studies conclude that the dipole moments of simple-metal clusters are very small and essentially consistent with zero value which characterizes the clusters as metallic.

Compared to simple metals, the coinage metals, $\mathrm{Ag}, \mathrm{Au}$, and $\mathrm{Cu}$, exhibit additional complexity due to the respective filled electronic d shells at $2 \mathrm{eV}(\mathrm{Au}, \mathrm{Cu})$ or $4 \mathrm{eV}(\mathrm{Ag})$ from the Fermi energy. They are relatively strongly localized around the atoms as compared to the free s electrons, but their polarizability plays an essential role, for instance, for the optical response of the materials, leading to the screening of the surface-plasmons in noble-metal clusters. ${ }^{6-9}$ Angular-momentum analysis of spherical noble-metal clusters reveals that a part of their orbitals are atomic-like "super-atom" states of largely delocalized electrons as they would also appear in a spherical jellium model, ${ }^{10}$ while another part essentially originating from the d electrons, does not show such behavior. Clearly, the delocalized states are expected to be rather well described by the jellium model, unlike the d states.

A direct consequence of the absence of an internal electric field in metals is that the creation of any cavity within a metal object in an external electric field will not change the overall polarization of the object; the cavity remains "invisible" to a given static external electric field. ${ }^{2}$ This motivates our investigation of compact noble-metal clusters in comparison with shells derived from them, in order to study the degree to which the clusters fulfill this criterion of metallicity. Two limiting cases are imaginable: a breakdown of screening, which would lead essentially to a dielectric-like polarizability proportional to the number of atoms, or the opposite, a complete screening by charge arrangements in the surface layer, which would render the existence of a cavity inconsequential.

Shell structures have been considered by Wang et al. ${ }^{11}$ who compared the polarization 

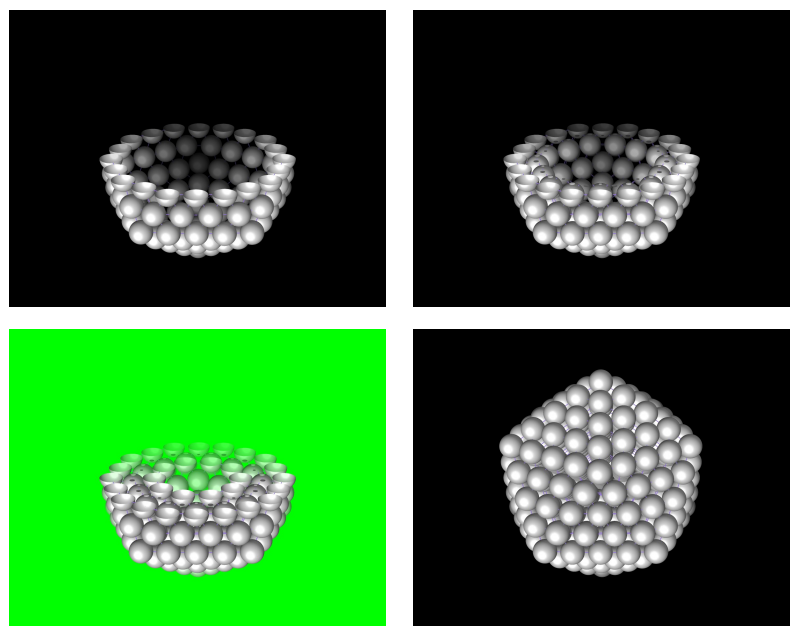

Figure 1: 309-atom compact icosahedral Ag cluster shown alongside the shell structure of 162,254 , and 296 atoms derived from it.

induced by a constant homogeneous electric field in different $\mathrm{Au}_{N}(N \leq 56)$ relaxed compact structures and cages. They reported, in particular, that the dipole moment per atom of the hollow cages increased roughly linearly with the number of atoms, whereas in the compact structures it remained essentially constant. Therefore, the total polarizability is roughly proportional to the volume for both geometries, as expected from the classical behaviour. In addition, Jellinek et al. considered site-specific polarizabilities to analyze the size dependence of induced dipole moments for simple-metal compact clusters. ${ }^{12,13}$

In the present work, we have taken a different and conceptually simpler approach to the problem of metallicity of metal clusters. To study the degree of metallic screening in noble-metal clusters, we also use the static dipole moment induced by a constant homogeneous electric field. However, we compare compact icosahedral clusters with shell structures derived from them by the consecutive removal of $1,13,55,147 \ldots$ atoms. These shell structures, shown in Fig. 1, are used without further ionic relaxation. In this way we can compare the sole effects of charge rearrangement on a very fine scale while excluding the effects of structural rearrangements or relaxations. This allows us to develop a fine analysis of the changes in the electronic density. For the interpretation of the results, this analysis is complemented by results for the well-known spherical jellium model. 


\section{Models and Methods}

Atomistic calculations: We use the well-known series of icosahedral clusters consisting of concentric atomic shells of $1,12,42,92,162 \ldots$ atoms, resulting in compact cluster sizes of $13,55,147,309,561 \ldots$ atoms. In particular, we focus on the 309-atom icosahedra. Starting from the relaxed structure for both $\mathrm{Au}$ and $\mathrm{Ag}$, we create shell structures by removing increasing numbers of atoms from the inside. This results in a 296-atom cage (three outer atomic shells, 13-atom core removed), a 254-atom cage (two outer atomic shells, having the 55-atom core removed) and the 162-atom cage (one outer atomic shell, having the 147-atom core removed). These shells are shown in Fig. 1. On purpose, we do not further relax the thus created cages in order to obtain exclusively the electronic effects, without the interference of structural relaxation effects. The relaxation of the compact 309-atom clusters was carried out using the VASP code. ${ }^{14,15}$ For comparison, we have also calculated the density for a compact $\mathrm{Na} 309$-atom cluster.

We then calculate both the ground state with a constant and homogeneous external electric field of $E(z)=0.2 \mathrm{eV} /(\mathrm{e} \AA)=0.00389 \mathrm{E}_{\mathrm{h}} /\left(\mathrm{ea}_{\mathrm{B}}\right)$, and the field-free ground state using the real-space code octopus. ${ }^{16,17}$ The field is applied along one of the five-fold symmetry axes in all cases. Norm-conserving Troullier-Martins pseudopotentials have been used which include the $d$ electrons in the valence, that is, with 11 valence electrons for each atom. The gradient-corrected PBE exchange-correlation potential has been used. The real-space grid spacing was set to $0.18 \AA$ for the silver, $0.20 \AA$ for the gold, and 0.30 $\AA$ for the sodium cluster. The calculation domain was chosen to be a sphere with a radius $5 \AA$ larger than the outermost atoms (with the cluster centered around the origin). All calculations have been carried out for the neutral systems.

Jellium calculations: We start from a compact $\mathrm{Na}_{N}$ jellium cluster of radius $R_{\text {ext }}=$ $r_{\mathrm{s}} N^{1 / 3}$, where $r_{\mathrm{s}}=4 \mathrm{a}_{\mathrm{B}}$. Then we create a series of neutral spherical cage structures of fixed external radius $R_{\text {ext }}$ and internal radius $R_{\text {int }}\left(N_{\mathrm{r}}\right)=\left(1-N_{\mathrm{r}} / N\right)^{1 / 3} R_{\text {ext }}$, where $N_{\mathrm{r}}$ is the number of remaining atoms. Although the electron orbitals exhibit a closed-shell configuration only for some values of $N_{\mathrm{r}}$, in order to keep the simplicity of the jellium 
model we assume that the electron density has spherical symmetry also for open-shell configurations. Thus, it is easy to analyze in detail the dependence of the static response on the size of the inner cage.

We considered two cases. The first one, $N=309\left(R_{\text {ext }} \simeq 1.43 \mathrm{~nm}\right)$ is the natural choice for comparison with our ab initio calculations since $\mathrm{Na}_{309}$ clusters exhibit the same icosahedral arrangement as $\mathrm{Ag}_{309} \cdot{ }^{18}$ The second is $N=338\left(R_{\text {ext }} \simeq 1.47 \mathrm{~nm}\right)$, the atomistic analogue of which shows neither symmetry nor energetic stability upon addition/removal of atoms. However, whereas jellium $\mathrm{Na}_{309}$ has the open-shell configuration $1 \mathrm{~s}^{2} 1 \mathrm{p}^{6} 1 \mathrm{~d}^{10} 2 \mathrm{~s}^{2} \ldots 4 \mathrm{p}^{3}$, the HOMO level of $\mathrm{Na}_{338}$ is fully occupied, $1 \mathrm{~s}^{2} 1 \mathrm{p}^{6} \ldots 4 \mathrm{p}^{6} 2 \mathrm{i}^{26}$ being the corresponding configuration.

If the external electric field is applied along the $z$ direction, the induced charge can be written as $\delta \rho(\mathbf{r})=\delta \rho_{r}(r) \cos \theta$, where $r$ is the radial coordinate and $\theta$ is the azimuthal angle. We evaluate $\delta \rho_{r}(r)$ by using well-established perturbation-theory techniques which exploit the spherical symmetry of the system. ${ }^{19-23}$ The induced dipole moment is simply proportional to the integral of $r^{3} \delta \rho_{r}(r)$.

\section{Results}

In Fig. 2, we show the dipole moment induced by a constant external electric field in icosahedral 309-atom gold and silver clusters along with the result for the 296-atom three-layer shell, the 254-atom two-layer shell, and the 162-atom one-layer shell. Clearly, already the polarization of only one atomic layer produces a dipole moment close to that of the compact structure; the difference is only about $4 \%$ for both gold and silver. What's more, the two-layer shells have values that are essentially equal to those of the compact clusters. Seen from the classical point of view, i.e., assuming that the induced densities are restricted to the surfaces, this seems to suggest that we have a classical metal which screens outer electric fields very well; only when the structure is reduced to as little as one atomic layer, the screening becomes less complete although, as indicated above, only relatively little. 


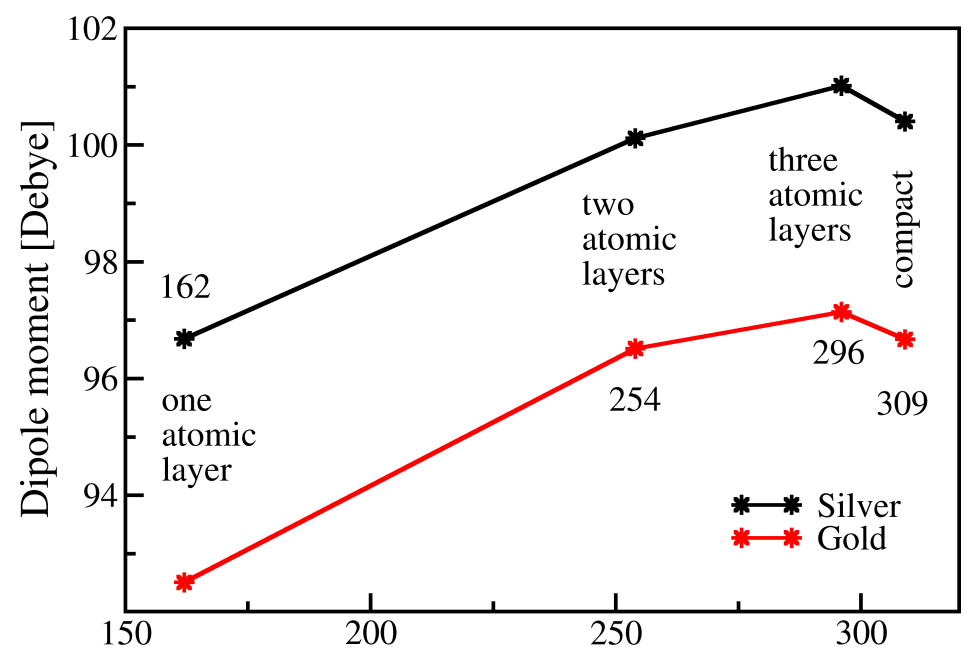

Figure 2: Induced dipole moments of icosahedral cage structures compared with the compact 309-atom clusters for gold and silver. A shell of one atomic layer thickness is enough to produce roughly $96 \%$ of the screening of the compact cluster. By contrast, the counter-intuitive increase for a small cavity (296 atoms) remains inexplicable in the classical picture.

However, it is immediately obvious that this picture cannot be complete. At 296 atoms, i.e., when only the 13-atom core is removed from the 309 compact cluster and three atomic layers constitute the shell structure, the polarizability increases as compared to the compact structure. From the classical point of view, this is wholly unexpected. Also, it is not restricted to the special case of the $\mathrm{I}_{h}$-309-derived clusters. Equivalent tendencies have been obtained for shell structures obtained from 147- and 55-atom compact icosahedra (see Supplementary Fig. S1).

In Fig. 3, we show the densities $\rho_{\mathrm{E}}-\rho_{\mathrm{GS}}$ induced by the external electric field for the compact $\mathrm{Ag}_{309}$ cluster, the 162-atom one-layer Ag cage, and a compact $\mathrm{Na}_{309}$ cluster for comparison. Each column shows different views of the same situation. First, the overall polarization in all cases has a dipole shape, with the values of the induced densities being largest at the surface. Naturally, they are smeared out compared to the classical picture of charges on abrupt surfaces. However, there are, in the sub-surface region, contributions of opposite sign, clearly visible through the transparent red iso-surfaces and also in the color-coded slab representations in the lower-most panel. In the Ag cluster, they are clearly centered around the atoms, hinting at an involvement of the d electrons acting 

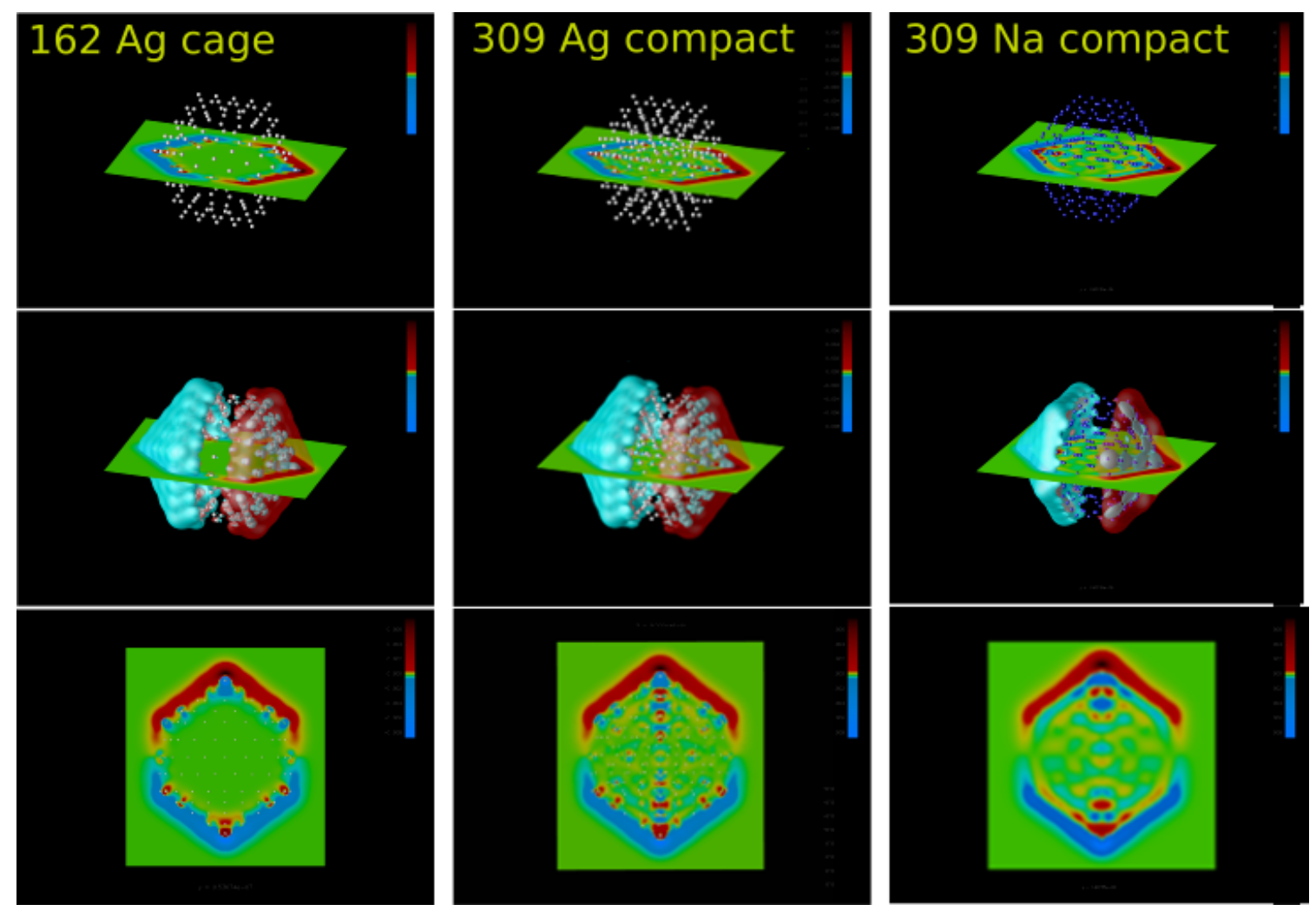

Figure 3: Induced densities of the 162-atom one-layer Ag cage (left panels) and the compact 309-atom Ag icosahedron. Shown are (upper panels) the geometric structure along with a color slab representation cutting through the center of the clusters with the induced density values as given by the color scale; middle panels: im addition, isosurfaces of the induced density, where the positive side (red) is partially transparent to bring out the structure below the surface; lower panels: the same color-coded slab representation as in the upper panels for better visibility. The panels on the right-hand side show the same for an (atomistic) $\mathrm{Na}_{309}$ cluster.

as "sensors" of local changes in fields and densities. In general, it is striking to see the degree to which the picture is similar for the one-atom-layer shell and the compact cluster. The small difference between them is best seen in the color-coded slab representations in the lower-most panels: inside the cage, as intuitively expected, there is essentially no contribution to the induced density, whereas in the compact cluster, there are clearly visible non-zero contributions spread out over the full volume. Also here, the subsurface regions of opposite sign as the adjacent surface are clearly visible. Nonetheless, the picture is not easily understood due to the presence of the d electrons and the resulting complexity of the induced densities.

Therefore, the induced densities of the simpler case of an atomistic 309-atom Na cluster are included in Fig. 3 (right column). They are similar to those for the noble 
metals, although the absence of d electrons simplifies the picture. The overall polarization is similar, with the main contribution to the induced charges at the surface and the subsurface regions of opposite sign. However, the atomic structure does not play a significant role here, in contrast to the noble metals where the d electrons act as "sensors" of the changes in density. The internal structure of the induced density is dictated more by the shape (facets, ...) than by the atomistic structure.

In order to obtain a physical understanding of the results of these atomistic calculations, we turn to the much simpler spherical jellium model. We consider sodium in order to keep the similarity with the one s electron per noble-metal atom, i.e., disregarding the $\mathrm{d}$ electrons in the counting. As indicated in the section Models and Methods, we consider two cases: a cluster corresponding to $N=309$ atoms, and a second case of similar "size" and number of atoms, $N=338$, which is an electronically closed-shell structure.

In Fig. 4, we show the dependence of the dipole moment on the number of atoms for the series of cage structures derived from jellium $\mathrm{Na}_{338}$ is discontinuous at $N_{\mathrm{r}}=330,298$, and 200. Inspection of the electron configurations shows that these are the values where the maximum $n$ quantum number of the occupied $n \ell$ levels, $n_{\mathrm{m}}$, changes. Such discontinuities also appear for the cage structures corresponding to jellium $\mathrm{Na}_{309}$ where, in addition, the dipole moment oscillates when $n_{\mathrm{m}}=3$ and has further smaller discontinuities when $n_{\mathrm{m}}=2$. These additional features are related to changes of the $\ell$ quantum number of the HOMO level; they are also present for the $\mathrm{Na}_{338}$-based structures but to a much lesser extent.

An oscillatory behavior of the static polarizability also appears for compact spherical jellium clusters when varying their radius $R_{\text {ext }} \cdot{ }^{21}$ Since the polarizability can be written as $\alpha=\left(R_{\text {ext }}+\delta\right)^{3}$, where $\delta$ is the electron-density spill-out parameter, ${ }^{24}$ the non-monotonic dependence of the polarizability on $R_{\text {ext }}$ can be attributed to trend changes of the density spill-out when a new level starts to be occupied. This explanation also holds for the present case with the sole exception that the number of occupied levels varies while keeping the size $R_{\text {ext }}$ of the system. This can be seen in Fig. 5 (a), where the radial term $\delta \rho_{r}(r)$ of the induced charge is shown as a contour map as a function of the number of 


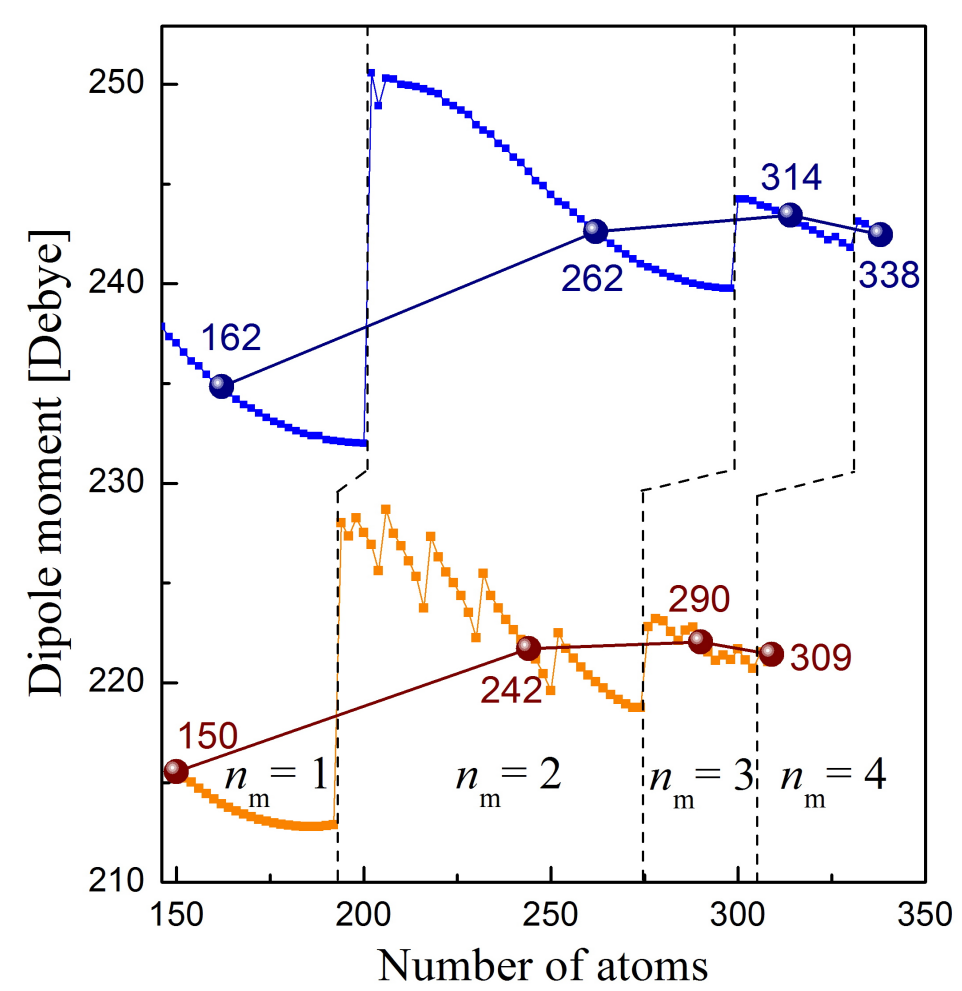

Figure 4: Induced dipole moment by an incident $\mathrm{E}(\mathrm{z})=0.2 \mathrm{eV} /(\mathrm{e} \AA)$ for $\mathrm{Na}_{338^{-}}$and $\mathrm{Na}_{309^{-}}$ based spherical jellium cage structures. The results for geometries mimicking structures with one, two, and three atomic shells are highlighted. The lines connecting the symbols are guides to the eye, highlighting the similarities with Fig. 2.

atoms of the $\mathrm{Na}_{338}$-based jellium cage structures (for $\mathrm{Na}_{309}$ the overall picture is the same but less well defined). The discontinuities of the polarizability are reflected by almost rigid displacements of the induced charge. In addition, the amplitude of the Friedel-like oscillations of the induced charge also changes abruptly.

To relate these results directly to the coinage-metal cage structures, we highlight in Fig. 4 the situations that resemble the most closely the atomistic cages. They correspond to a partitioning of the compact jellium background into a core surrounded by three concentric layers, cf., Fig. 5(b). Although the width $d$ of the latter is somewhat arbitrary, we choose $d=0.29 \mathrm{~nm}$ based on the the geometry of the (atomistic) icosahedral $\mathrm{Na}_{309}$ cluster. Removing consecutively the core and the first and second layers, we obtain three cage structures corresponding to $N=314,262$, and 162 atoms, respectively, if we start from $\mathrm{Na}_{338}$. For the compact $\mathrm{Na}_{309}$ jellium cluster, we get $N=290,242$, and 150, fairly close to the icosahedral series $(296,254,162)$. The dipole moments for these selected geometries are highlighted in Fig. 4, and we see that they follow the same 


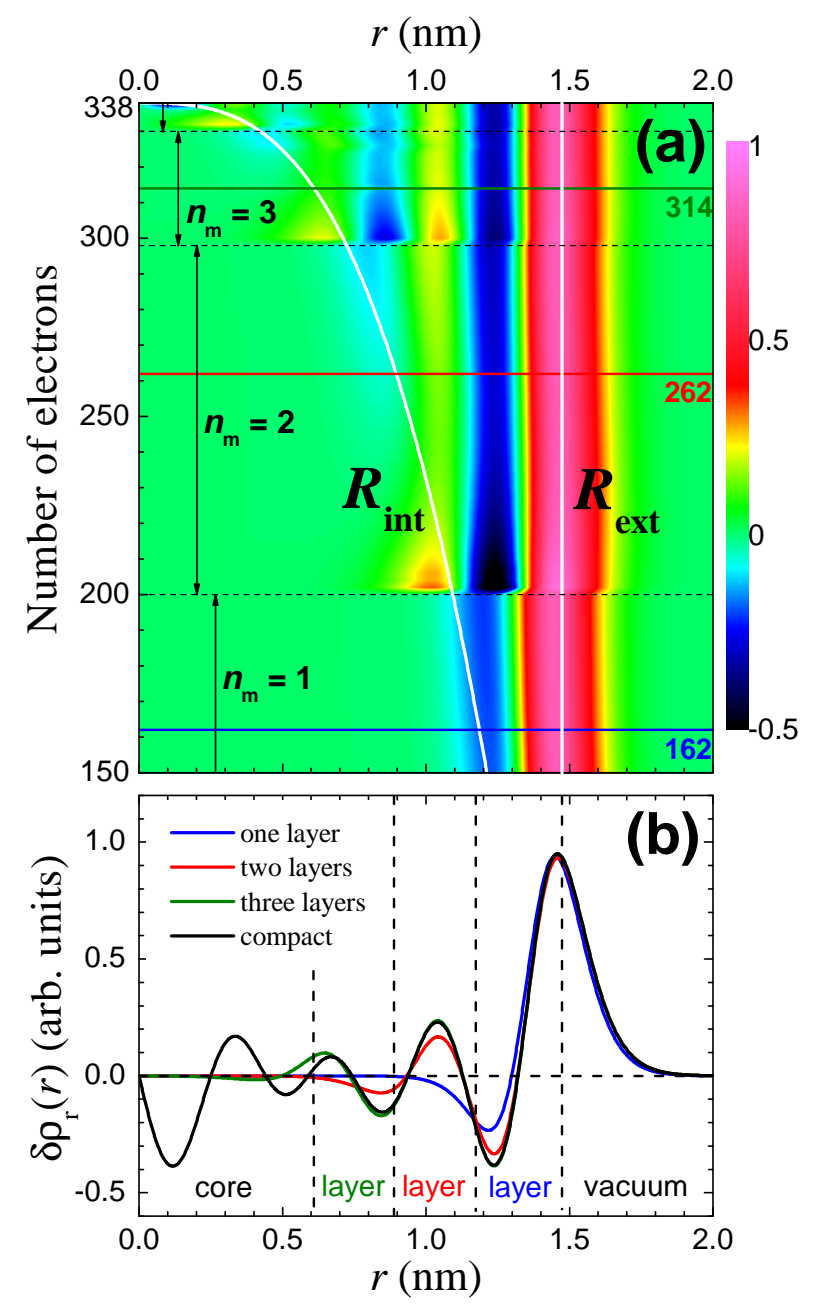

Figure 5: Radial part $\delta \rho_{r}(r)$ of the induced densities for $\mathrm{Na}_{338}$-based spherical jellium cage structures. Panel (a): contour map showing the overall dependence on the number of atoms. The white lines indicate the values of $R_{\text {int }}$ and $R_{\text {ext }}$. Panel (b): comparison between the induced densities for the compact and for selected cage structures (see text).

behavior as the coinage-metal cage structures presented in Fig. 2: the polarizability of the clusters remains approximately constant if there are at least two atomic layers in the cage structure, with only small variations of less than one percent. These small variations are not monotonic due to the discrete nature of the electron energy levels, thus explaining the local maximum of the dipole moment upon creation of small cavities in either the real, atomistic clusters or the jellium models thereof. Finally, in the limit of a one-atom shell thickness, the polarizability is reduced by a mere $3-4 \%$, consistent with the result of the Ag and Au shells, indicating almost complete screening even by one layer of atoms.

The induced charge around the outer surface is practically the same for the compact $\mathrm{Na}_{338}$ cluster and for the $\mathrm{Na}_{316}$ and $\mathrm{Na}_{264}$ cage structures (see Fig. 5(b)). In all the 
cases, the main contribution is located directly at the surface, explaining the high degree of similarity of the induced dipole moments of compact clusters and shells. The Friedellike oscillations lead to ranges of $\rho_{\text {ind }}(r)$ of opposite sign as the surface charge, which is entirely consistent with the corresponding regions in the noble-metal structures and the compact $\mathrm{Na}_{309}$ cluster in Fig. 3. The creation of the cavities leads to a logical depletion of the Friedel-like oscillations of the induced charge because of the reduced spatial extension of the jellium background. Only for the one-atom thickness jellium- $\mathrm{Na}_{164}$, a very weak change of the induced density with respect to that of $\mathrm{Na}_{338}$ can be observed: the negative peak is less intense, and it is slightly shifted towards the interior. In addition, there are no further oscillations of the induced density due to the quasi two-dimensional character of the one-atom layer. In the Supplementary Information we include further results for the jellium model as well as a direct comparison with the atomistic Na structure.

\section{Conclusions}

By the measure we studied, i.e., the screening as evidenced by the dipole moment induced by a static external electric field in clusters with internal cavities, the behavior of quantum-sized noble-metal clusters is rather close to that of a classical perfect metal: one atomic shell suffices to endow a cage with about $96 \%$ of the polarizability of the compact cluster, two shells obtain $\approx 100 \%$. This indicates very efficient screening.

However, an increase of the induced dipole moment upon creation of a small cavity inside the cluster indicates quantum effects that are incompatible with the classical behavior, as is the distribution of the induced density. These effects have been reproduced using the spherical jellium model. This shows that, in addition to being "very metallic," the noble-metal clusters are both influenced by quantum effects and rather "jellium-like", notwithstanding the complicated electronic structure with the presence of the d electrons. The latter do, however, act as "sensors" which witness the residual fields and density changes inside the clusters, around the positions of the atoms.

The discontinuous, non-monotonous change of the static polarizability is due to shell- 
closing effects, similar to the discontinuities in the size-dependence of the polarizability of compact spherical clusters. The atomistic shell structures then happen to lie on somewhat arbitrary points of the complicated dipole-vs.- $\mathrm{N}_{\text {atoms }}$ curve because atomistic and electronic shell closures do not, in general, occur at the same numbers of atoms. The changes of the induced dipole moment and their explanation are consistent for all, the $\mathrm{Au}$ and $\mathrm{Ag}$ clusters of different sizes and the jellium model.

We note that these results show that the induced dipole moment per atom is not a useful measure if compact and cage structures are compared, as it is clear from the fact that the 162-atom shell and the 309-atom compact cluster have almost identical induced dipole moments which are, however, very different from that of a 162-atom compact cluster. Finally, we emphasize that the present discussion applies to the static case; the dynamic response of metal clusters and shells as well as the role of the d electrons will be qualitatively different.

In conclusion, the noble-metal clusters at a size of about 300 atoms, while being metallic, are still rather strongly affected by quantum effects, viz., electron shell closings and the non-homogeneous Friedel-like oscillations of the density.

\section{Acknowledgement}

We are indebted to Robert L. Whetten and Xóchitl López Lozano for stimulating discussions that germinated this research. We also acknowledge enlightening discussions with Antonio Fernández-Dominguez. This work has been carried out thanks to the support of the $A^{*}$ MIDEX grant ( ${ }^{\circ}$ ANR-11-IDEX-0001-02) funded by the French Government "Investissements d'Avenir" program. We acknowledge support from the French National Research Agency (Agence Nationale de Recherche, ANR) in the frame of the project "FIT SPRINGS", ANR-14-CE08-0009. This work has used HPC resources from GENCIIDRIS (Grant 2017-096829). Moreover, HCW and RSR would like to acknowledge the contribution of the International Research Network IRN Nanoalloys (CNRS). PGG ac- 
knowledges funding from the Spanish Government through the MICINN research grant RTI2018-099737-B-100 and the "María de Maetzu" Programme for Units of Excellence in R\&D (MDM-2014-0377).

\section{Conflict of interest}

There are no conflicts of interest to declare.

\section{References}

(1) Ball, P. The Smallest Metals. Nature Materials 2011, 10, 175.

(2) Jackson, J. D. Classical electrodynamics; 2nd ed.; Wiley: New York, NY, 1975.

(3) Bowlan, J.; Liang, A.; de Heer, W. A. How Metallic are Small Sodium Clusters? Phys. Rev. Lett. 2011, 106, 043401.

(4) Aguado, A.; Largo, A.; Vega, A.; Balbás, L. C. On the electric dipole moments of small sodium clusters from different theoretical approaches. Chemical Physics 2012, $399,252-257$.

(5) Gotz, D. A.; Shayeghi, A.; Johnston, R. L.; Schwerdtfeger, P.; Schafer, R. Structural evolution and metallicity of lead clusters. Nanoscale 2016, 8, 11153-11160.

(6) Liebsch, A. Surface-plasmon dispersion and size dependence of Mie resonance: Silver versus simple metals. Phys. Rev. B 1993, 48, 11317-11328.

(7) Serra, L.; Rubio, A. Core Polarization in the Optical Response of Metal Clusters: Generalized Time-Dependent Density-Functional Theory. Phys. Rev. Lett. 1997, 78, $1428-1431$.

(8) Cottancin, E.; Celep, G.; Lermé, J.; Pellarin, M.; Huntzinger, J.; Vialle, J.; Broyer, M. Optical Properties of Noble Metal Clusters as a Function of the Size: 
Comparison between Experiments and a Semi-Quantal Theory. Theoretical Chemistry Accounts: Theory, Computation, and Modeling (Theoretica Chimica Acta) 2006, 116, 514 .

(9) Weissker, H.-C.; López-Lozano, X. Surface plasmons in quantum-sized noble-metal clusters: TDDFT quantum calculations and the classical picture of charge oscillations. Phys. Chem. Chem. Phys. 2015, 17, 28379-28386.

(10) Jena, P.; Sun, Q. Super Atomic Clusters: Design Rules and Potential for Building Blocks of Materials. Chemical Reviews 2018, 118, 5755-5870, PMID: 29812916.

(11) Wang, J.; Yang, M.; Jellinek, J.; Wang, G. Dipole polarizabilities of medium-sized gold clusters. Phys. Rev. A 2006, 74, 023202.

(12) Jackson, K.; Yang, M.; Jellinek, J. Site-Specific Analysis of Dielectric Properties of Finite Systems. The Journal of Physical Chemistry C 2007, 111, 17952-17960.

(13) Ma, L.; Jackson, K. A.; Wang, J.; Horoi, M.; Jellinek, J. Investigating the metallic behavior of Na clusters using site-specific polarizabilities. Phys. Rev. B 2014, 89, 035429 .

(14) Kresse, G.; Furthmüller, J. Efficiency of ab-initio total energy calculations for metals and semiconductors using a plane-wave basis set. Comput. Mat. Sci. 1996, 6, 15.

(15) Kresse, G.; Joubert, D. From ultrasoft pseudopotentials to the projector augmentedwave method. Phys. Rev. B 1999, 59, 1758.

(16) Marques, M. A. L.; Castro, A.; Bertsch, G. F.; Rubio, A. Comp. Phys. Comm. 2003, 151, 60 .

(17) Castro, A.; Marques, M. A. L.; Appel, H.; Oliveira, M.; Rozzi, C.; Andrade, X.; Lorenzen, F.; Gross, E. K. U.; Rubio, A. Phys. Stat. Sol. (b) 2006, 243, 2465.

(18) Noya, E. G.; Doye, J. P. K.; Wales, D. J.; Aguado, A. Geometric magic numbers of sodium clusters: Interpretation of the melting behaviour. Eur. Phys. J. D 2007, $43,57-60$. 
(19) Ekardt, W. Work function of small metal particles: Self-consistent spherical jelliumbackground model. Phys. Rev. B 1984, 29, 1558-1564.

(20) Ekardt, W. Dynamical Polarizability of Small Metal Particles: Self-Consistent Spherical Jellium Background Model. Phys. Rev. Lett. 1984, 52, 1925-1928.

(21) Puska, M. J.; Nieminen, R. M.; Manninen, M. Electronic polarizability of small metal spheres. Phys. Rev. B 1985, 31, 3486-3495.

(22) Prodan, E.; Nordlander, P. Structural Tunability of the Plasmon Resonances in Metallic Nanoshells. Nano Letters 2003, 3, 543-547.

(23) Varas, A.; García-González, P.; Feist, J.; García-Vidal, F. J.; Rubio, A. Quantum plasmonics: from jellium models to ab intitio calculations. Nanophotonics 2016, 5, 409-426.

(24) de Heer, W. A. The physics of simple metal clusters: experimental aspects and simple models. Rev. Mod. Phys. 1993, 65, 611-676. 
Graphical TOC Entry

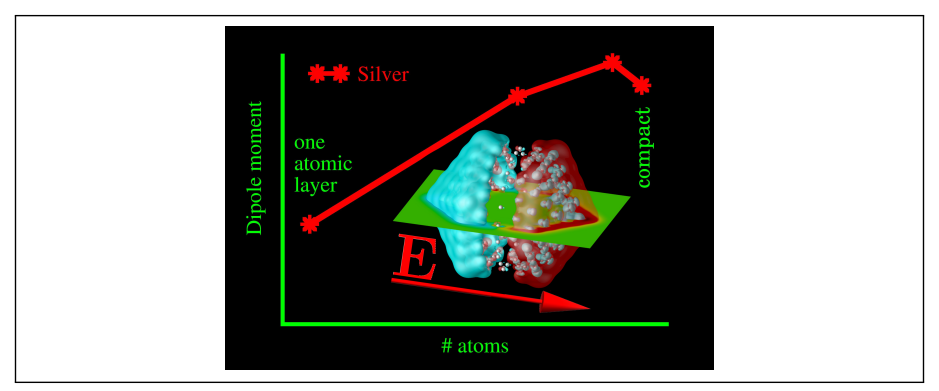

